\title{
ON THE EXISTENCE OF SPECIFIED CYCLES IN COMPLEMENTARY GRAPHS ${ }^{1}$
}

\author{
BY GARY CHARTRAND ${ }^{2}$ AND SEYMOUR SCHUSTER ${ }^{3}$
}

Communicated by Victor Klee, June 1, 1971

It is well known that in any gathering of six people, there are three people who are mutual acquaintances or three people who are mutual strangers. This statement has the graph-theoretic formulation that for any graph $G$ of order 6 , either $G$ or its complement $\bar{G}$ has a triangle. Furthermore, this statement is not true in general if "six" is replaced by a smaller integer.

The Ramsey number $r(m, n)$ may be considered a generalization of the above statement. For integers $m, n \geqq 2$, the number $r(m, n)$ is defined as the least integer $p$ such that for any graph $G$ of order $p$, either $G$ contains the complete subgraph $K_{m}$ of order $m$ or $\bar{G}$ contains $K_{n}$. Hence, $r(3,3)=6$. It is a trivial observation that $r(m, n)=r(n, m)$, and $r(2, n)=n$ for all $n \geqq 2$. Despite the fact that a great deal of research has been done on Ramsey numbers, only six values $r(m, n)$ have been determined for $m, n \geqq 3$ (see [1]); namely, $r(m, n)$ is known (for $m, n \geqq 3)$ only when $(m, n)=(3,3),(3,4),(3,5),(3,6),(3,7)$, $(4,4)$. Thus, no general formula for $r(m, n)$ has been determined for a fixed $m \geqq 3$ and arbitrary $n$; indeed, no such formula has even been conjectured.

There is a generalization of the problem of the three acquaintances and three strangers which is different from that which leads to the Ramsey numbers but which is just as natural. If we denote an $n$-cycle by $C_{n}$, then the above problem may be stated as: Given a graph $G$ of order 6 , either $G$ or $\bar{G}$ contains $C_{3}$. This suggests the following generalization. For $m, n \geqq 3$, the number $c(m, n)$ is defined as the least integer $p$ such that for any graph $G$ of order $p$, either $G$ contains $C_{m}$ or $\bar{G}$ contains $C_{n}$. Of course, $c(3,3)=6$. We wish now to announce formulas for $c(3, n), c(4, n)$, and $c(5, n)$ for all $n \geqq 3$.

THEOREM 1. If $n \geqq 3$, then

$$
\begin{aligned}
c(3, n) & =6 & & \text { if } n=3, \\
& =2 n-1 & & \text { if } n \geqq 4 .
\end{aligned}
$$

AMS 1969 subject classifications. Primary 0540.

Key words and phrases. Graph, cycle, Ramsey number, complement.

1 Partially supported by NSF Science Faculty Fellowship.

${ }^{2}$ Research supported in part by the Office of Naval Research.

3 Research supported by the National Science Foundation. 
OUtLINE OF PROOF. We have already noted that $c(3,3)=6$. That $c(3, n)=2 n-1$ for $n \geqq 4$ is verified by employing induction on $n$. The number $c(3,4)=7$ is established individually. Assume $c(3, n)=2 n-1$ for some fixed $n \geqq 4$ and consider the number $c(3, n+1)$. (For $m, n \geqq 1$, denote by $K(m, n)$ the complete bipartite graph of order $m+n$ whose vertex set may be partitioned as $V_{1} \cup V_{2}$, where $\left|V_{1}\right|=m$ and $\left|V_{2}\right|=n$ and where $e=u v$ is an edge if and only if $u \in V_{i}$ and $v \in V_{j}, i \neq j$. If $G_{1}$ and $G_{2}$ are connected graphs, then $G_{1} \cup G_{2}$ represents the disconnected graph with components $G_{1}$ and $G_{2}$.) If $H=K(n, n)$ so that $\bar{H}=K_{n} \cup K_{n}$, then $H$ has no 3 -cycle and $\bar{H}$ has no $(n+1)$-cycle; thus, $c(3, n+1) \geqq 2 n+1$.

Let $G$ be a graph of order $2 n+1$, and assume $G$ has no 3-cycle. Since $c(3, n)=2 n-1, \bar{G}$ contains an $n$-cycle $C: u_{1}, u_{2}, \cdots, u_{n}, u_{1}$. Denote the remaining vertices of $\bar{G}$ (and hence $G$ ) by $v_{1}, v_{2}, \cdots, v_{n+1}$. If any $v_{i}$ is adjacent in $\bar{G}$ to two consecutive vertices of $C$, then $\bar{G}$ contains an $(n+1)$-cycle, completing the proof. Suppose, then, that no such $v_{i}$ exists. We consider two cases.

Case 1. Assume there exist two alternate vertices of $C$, say $u_{j}$ and $u_{j+2}$, which are respectively joined in $\bar{G}$ to two distinct $v_{i}$.

Case 2. Assume no two alternate vertices of $C$ are respectively joined in $\bar{G}$ to distinct vertices $v_{i}$.

In each case, it can be shown that $\bar{G}$ contains an $(n+1)$-cycle, thereby proving that $c(3, n+1)=2 n+1$.

In the case of the numbers $c(4, n)$, there are two special cases to be considered, namely $c(4,4)$ and $c(4,5)$.

THEOREM 2. If $n \geqq 4$, then

$$
\begin{aligned}
c(4, n) & =6 & & \text { if } n=4, \\
& =7 & & \text { if } n=5, \\
& =n+1 & & \text { if } n \geqq 6 .
\end{aligned}
$$

OUTLINE OF PROOF. The numbers $c(4,4)$ and $c(4,5)$ are treated separately. To prove $c(4, n)=n+1$ for $n \geqq 6$, we use induction on $n$, with $c(4,6)=7$ verified first. We make the standard induction hypothesis, and consider $c(4, n+1)$ for some $n \geqq 6$. For $H=K(1, n)$ and $\bar{H}=k_{1} \cup K_{n}$, we observe that $H$ has no cycles and $\bar{H}$ no $(n+1)$ cycles so that $c(4, n+1) \geqq n+2$.

Let $G$ be a graph of order $n+2$ having no 4 -cycles. Because $c(4, n)=n+1, \bar{G}$ has an $n$-cycle $C$. Let $v_{1}$ and $v_{2}$ be the two vertices of $\bar{G}$ not on $C$. We may assume that neither $v_{1}$ nor $v_{2}$ is joined in $\bar{G}$ to two consecutive vertices of $C$ so that each of $v_{1}$ and $v_{2}$ is joined in $G$ to at 
least $\left\{\frac{1}{2} n\right\}$ vertices of $C$. (For a real number $x,\{x\}$ is the least integer not less than $x$.)

If $v_{1}$ and $v_{2}$ are mutually adjacent in $G$ to two or more vertices of $C$, then $G$ contains a 4 -cycle, which produces a contradiction. We then consider two cases depending on whether $v_{1}$ and $v_{2}$ are mutually adjacent to no vertices or one vertex of $C$. In either case, one can establish the existence of an $(n+1)$-cycle in $\bar{G}$, concluding the proof.

The formula for $c(5, n), n \geqq 5$, presents no exceptional cases.

THEOREM 3. If $n \geqq 5$, then

$$
c(5, n)=2 n-1 \text {. }
$$

Outline of PROOF. Again we employ mathematical induction with $c(5,5)=9$ handled separately. Assume $c(5, n)=2 n-1$ for some $n \geqq 5$, and consider $c(5, n+1)$. The graph $H=K(n, n)$ has no 5 -cycle and its complement $\bar{H}=K_{n} \cup K_{n}$ has no $(n+1)$-cycle, so that $c(5, n+1)$ $\geqq 2 n+1$. Let $G$ be a graph of order $2 n+1$ possessing no 5 -cycle. Since $c(5, n)=2 n-1$, we have the existence of an $n$-cycle $C: u_{1}$, $u_{2}, \cdots, u_{n}, u_{1}$ in $\bar{G}$. If any of the remaining vertices $v_{1}, v_{2}, \cdots, v_{n}$, and $v_{n+1}$ is adjacent in $\bar{G}$ to two consecutive vertices of $C$, then $\bar{G}$ has an $(n+1)$-cycle, so we assume this is not the case. If the vertices $v_{i}$ induce $K_{n+1}$ in $\bar{G}$, then $\bar{G}$ has an $(n+1)$-cycle. Thus, we assume some two distinct $v_{i}$, say $v_{1}$ and $v_{2}$, are adjacent in $G$. Three cases are then treated according to the manner in which $v_{1}$ and $v_{2}$ are joined to the vertices of $C$.

Case 1 . Assume there is a vertex $v_{k}(k \neq 1,2)$ such that $v_{1}$ and $v_{k}$ are joined in $G$ to a vertex $u_{i}$ on $C$, and $v_{2}$ and $v_{k}$ are joined in $G$ to a vertex $u_{j}$ on $C$. Here a 5-cycle in $G$ is produced regardless of whether $u_{i}$ and $u_{j}$ are distinct. (If $n$ is odd, Case 1 necessarily applies. Hence, $n$ is even in the subsequent cases.)

Case 2. Assume Case 1 does not hold and there exists some vertex $v_{k}(k \neq 1,2)$ which is adjacent in $G$ to no vertex of $C$ which is joined in $G$ to $v_{1}$ or $v_{2}$. In this case, the existence of an $(n+1)$-cycle in $\bar{G}$ is established.

Case 3. Assume that Case 1 and Case 2 do not hold. This implies that each $v_{k}, k \neq 1,2$, has the properties that whenever $v_{1} u_{i}$ and $v_{k} u_{i}$ are in $G$, then $v_{2} u_{i}$ is in $\bar{G}$, and whenever $v_{2} u_{j}$ and $v_{k} u_{j}$ are in $G$, then $v_{1} u_{j}$ is in $\bar{G}$. Here we show that either $v_{1} v_{k}$ or $v_{2} v_{k}$ is an edge of $G$, for each $k \geqq 3$, and that we are under the conditions of Case 2, where the roles of $v_{1}$ and $v_{2}$ are played by either $v_{1}$ and $v_{k}$ or by $v_{2}$ and $v_{k}$. Hence, an $(n+1)$-cycle of $\bar{G}$ exists here also and the proof is complete. 
Further, the number $c(6,6)$ can be shown to have the value 8 . The argument we have constructed is quite lengthy and will be published elsewhere, together with the full details of the proofs of Theorems 1-3.

\section{REFERENCES}

1. J. E. Graver and J. Yackel, Some graph theoretic results associated with Ramsey's tneorem, J. Combinatorial Theory 4 (1968), 125-175. MR 37 \#1278.

Western Michigan University, Kalamazoo, Michigan 49001

University of California, Santa Barbara, California 93106 